\title{
A curated target gene pool assisting disease prediction and patient-specific biomarker selection for lung squamous cell carcinoma
}

\author{
BIN HUANG $^{1 *}$, NING ZHONG ${ }^{2 *}$, HONGBAO CAO $^{3,4}$ and GUIPING YU ${ }^{5}$ \\ ${ }^{1}$ Department of Cardiothoracic Surgery, Affiliated Jiangyin Hospital of Southeast University, Jiangyin, Jiangsu 214400; \\ ${ }^{2}$ Department of Cardiothoracic Surgery, The First People's Hospital of Kunshan, Kunshan, Jiangsu 215300, P.R. China; \\ ${ }^{3}$ Department of Genomics Research, R\&D Solutions, Elsevier Inc., Rockville, MD 20852; ${ }^{4}$ Unit on Statistical Genomics, \\ National Institute of Mental Health/National Institute of Health, Bethesda, MD 20892, USA; \\ ${ }^{5}$ Department of Cardiothoracic Surgery, The Affiliated Jiangyin Hospital of Southeast University \\ Medical College, Jiangyin, Jiangsu 214400, P.R. China
}

Received December 6, 2017; Accepted June 13, 2018

DOI: $10.3892 / \mathrm{ol} .2018 .9241$

\begin{abstract}
There have been hundreds of genes demonstrated to be associated with lung squamous cell carcinoma (LSCC), presenting various degrees of association with this disease. In the present study, gene vectors were investigated as genetic biomarkers for the diagnosis and personalized treatment of LSCC. A LSCC genetic database (LSCC_GD) was developed through literature-associated data analysis, where 260 LSCC target genes were curated. Subsequently, numerous associations between these genes and LSCC were studied. Following this, a sparse representation-based variable selection (SRVS) was employed for gene selection from two LSCC gene expression datasets, followed by a case/control classification. Results were compared using analysis of variance (ANOVA)-based gene selection approaches. Using SRVS, a gene vector was selected from each dataset, resulting in significantly higher classification accuracy (CR), compared with randomly selected genes (For datasets GSE18842 and GSE1987, CR=100 and $100 \%$ and permutation $\mathrm{P}=5.0 \times 10^{-4}$ and $1.8 \times 10^{-3}$, respectively). The SRVS method outperformed ANOVA in terms of the classification ratio. The results indicated that, for a given dataset, there may be a gene vector from the 260 curated LSCC genes that possesses significant prediction power. SRVS is effective
\end{abstract}

Correspondence to: Dr Guiping Yu, Department of Cardiothoracic Surgery, The Affiliated Jiangyin Hospital of Southeast University Medical College, 163 Shoushan Road, Jiangyin, Jiangsu 214400, P.R. China

E-mail: xiaoyuer97103@163.com

${ }^{*}$ Contributed equally

Key words: lung squamous cell carcinoma, non-small-cell lung carcinoma, sparse representation, variable selection in identifying the optimum gene subset target for personalized treatment.

\section{Introduction}

Lung cancer is the leading cause of cancer-associated mortalities worldwide (1). There are two main types of lung cancer, small-cell lung carcinoma (SCLC) and non-SCLC (NSCLC), and the latter accounts for $\sim 84 \%$ of all lung cancer cases in USA in 2018 (2). Lung squamous cell carcinoma (LSCC) is a subtype of NSCLC, and has a pathogenesis that is closely correlated with a history of tobacco smoking (3). Despite notable advances in the targeted treatment of patients with NSCLC, patients with LSCC do not benefit from these major improvements. For example, patients with the adenocarcinoma subtype of NSCLC are most likely to respond to epidermal growth factor receptor (EGFR) kinase inhibitors; however, patients with LSCC rarely respond to these EGFR kinase inhibitors (4). Recent studies have identified genes that may serve important roles in LSCC $(5,6)$; however, the molecular abnormalities and genetic mechanics of LSCC remain primarily unknown. Therefore, significant research into the genetic causes of LSCC has been undertaken, targeting earlier diagnosis and novel treatment development for the disease.

There have been hundreds of genes associated with LSCC, reflecting its heterogeneity. Mutations of a number of risk genes have been frequently reported in LSCC, such as tumor protein P53 (TP53) (7); however, these genes may also be biomarkers for multiple other diseases. For example, TP53 may serve as the diagnostic marker for SCLC (7) and chronic lymphocytic leukemia (8). This decreases the specificity of these genes as biomarkers for the diagnosis and treatment of LSCC; however, a number of genes only appear in a limited portion of LSCC cases, such as ACKR3 and ADGRL2 $(9,10)$. Furthermore, there are numerous novel genes identified each year for LSCC. For example, in 2017, fibroblast growth factor receptor (FGFR) 4 and microRNA 145 were novelly reported as LSCC risk genes that serve roles in the mechanism of the disease $(11,12)$. These 
genes have been identified in limited LSCC studies, which may be due to the specificity of genome variations in different patients (13); therefore, early diagnosis/prediction of LSCC may require multiple genes as biomarkers. Furthermore, patient-specificity should also be taken into account when selecting the appropriate individualized treatment.

To address this issue, the present study first developed a LSCC genetic database (LSCC_GD), curating all LSCC target genes available within Pathway Studio (http://pathwaystudio. com), a literature-based pathway analysis tool used to model associations between proteins, genes, complexes, cells, tissues and diseases. It has been demonstrated that Pathway Studio possesses the largest real-time updated databases in this field of study (14). The disease prediction capability of these curated genes within LSCC_GD was tested using multiple independent gene expression datasets, where the selected genes were used to classify patients with LSCC from healthy controls (LSCC case/control classification). A sparse representation-based variable selection (SRVS) algorithm was employed to select the optimum gene vectors for a given patient group as biomarkers/features to achieve the highest case/control classification accuracy. Cao et al (15) demonstrated the effectiveness of the SRVS algorithm in genetic and imaging variable selection. Instead of selecting a specific number of variables, the SRVS method generates a sparse regression weight for each variable, which can be used for variable ranking.

The results confirmed the specificity of the genomic variation of different groups of patients with LSCC, and supported the hypothesis that for a given group of patients with LSCC, there is a gene vector, from the curated LSCC target genes, that possesses significant predication power to distinguish LSCC cases from healthy controls.

\section{Materials and methods}

Development of LSCC_GD. The LSCC_GD database contains 260 genes (LSCC_GD $\rightarrow$ Related Genes). These genes were identified from previously demonstrated associations with LSCC, which are supported by 685 references (LSCC $\rightarrow$ Ref for Disease-Gene Relation). The LSCC_GD database also includes 56 drugs (LSCC_GD $\rightarrow$ Related Drugs), 101 diseases (LSCC_ $\mathrm{GD} \rightarrow$ Related Diseases) and 100 pathways (LSCC_GD $\rightarrow$ Related Pathways) associations with LSCC. These LSCC-associated entities were acquired using Pathway Studio. LSCC_GD also includes the information of 658 and 126 supporting references for LSCC-Gene and LSCC-Drug associations, respectively. For each association, there are $\geq 1$ supporting references. The reference information includes the title of and the associated sentences from the source where an association was identified. The current LSCC_GD database has been deposited into the 'Bioinformatics Database' (http://gousinfo.com/database/Data Genetic/LSCC_GD.xlsx). It is scalable and will be updated monthly or upon request. Fig. 1 presents the database schema of the curated database LSCC_GD.

Gene Set Enrichment Analysis (GSEA) and Sub-Network Enrichment Analysis (SNEA) were conducted to identify pathways, diseases, and drugs (small molecules) associated with the 260 LSCC genes. Fisher's exact test was employed for GSEA and SNEA to measure the gene-enrichment in annotation terms and the significance of the overlap between a selected gene
Table I. Statistics of two gene expression datasets.

\begin{tabular}{lcc}
\hline NCBI GEO ID & GSE18842 & GSE1987 \\
\hline \#LSCC case/control & $46 / 45$ & $17 / 9$ \\
\#Genes from LSCC_GD & 232 & 702 \\
\hline
\end{tabular}

NCBI, National Center for Biotechnology Information; LSCC, lung squamous cell carcinoma.

group and a given pathway/sub-network. The top 100 pathways (LSCC $\rightarrow$ Related Pathways) were acquired using the Pathway Enrichment Analysis (PEA) module of Pathway Studio. The 260 LSCC genes were significantly enriched within these pathways $\left[\mathrm{P}<4.4 \times 10^{-05} ; \mathrm{q}=0.001\right.$ for false discovery rate $\left.(\mathrm{FDR})\right]$. The 101 diseases (LSCC $\rightarrow$ Related Diseases) were identified using the SNEA module (http://pathwaystudio.gousinfo.com/SNEA.pdf). The 260 LSCC target genes were significantly overlapped with the genes associated with each of these 101 diseases $\left(\mathrm{P}<1.9 \times 10^{-33}\right.$; $\mathrm{q}=0.001$ for FDR). A number of the pathways and diseases have been previously implicated in LSCC, indicating the pathological association between these target genes and LSCC.

The Gene-Gene Interaction (GGI) network (LSCC $\rightarrow$ GGI Network) was generated based on the enriched pathways. Two genes were identified as connected if they shared $\geq 1$ pathways. The number of shared pathways was the edge weight. A 7-by-7 GGI network was presented as an example, and the full network was presented in the form of an adjacent matrix in the LSCC $\rightarrow$ GGI Network. The 105 potential drugs (LSCC $\rightarrow$ Potential Drugs) were identified using the SNEA module of Pathway Studio. The drugs/small molecules were significantly associated with the LSCC target genes, and the majority of these have not been identified in clinical trial (92/105). The identified drugs/small molecules may represent potential drug candidates for LSCC. It is notable that these drugs demonstrated significant overlap with the drugs/small molecules associated with LSCC directly $\left(\right.$ LSCC_GD $\rightarrow$ Related drugs; $\mathrm{P}=1.31 \times 10^{-18}$ ).

SRVS for gene vector selection. The SRVS algorithm (15) was used to rank the 260 LSCC target genes according to a given experiment dataset. For each gene, a sparse weight will be assigned by SRVS. The gene vector composed of the top $\mathrm{n}$ genes ranked by SRVS will be the genetic marker for the LSCC case/control classification, where $n$ is the number of genes corresponding to the maximum classification ratio (CR) as defined as:

$$
C R=\frac{\# \text { correctly classified } \text { subjects }}{\# \text { total subjects }}
$$

The premise of the SRVS algorithm is to select an optimum number of features, according to sparse representation theory, when there were notably more features than samples. In the present study, the figures to be selected were genes. The input of the SRVS algorithm were the values of gene expression, and the output were the weight for each gene. These weights were defined as the SRVSScore, which was used to rank the genes. 


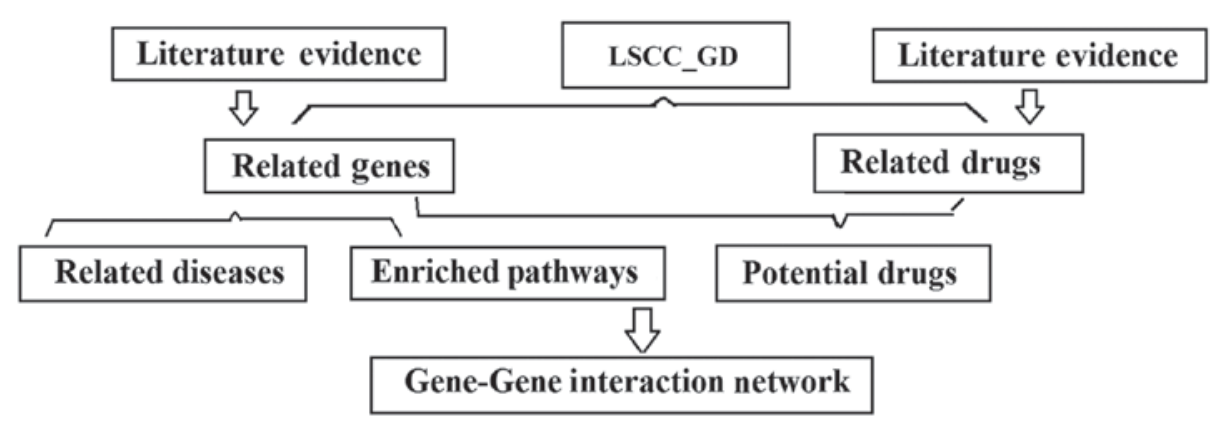

Figure 1. The LSCC_GD database schema. LSCC associated genes, drugs were collected from literature data mining. LSCC associated pathways were collected from Gene Set Enrichment Analysis, based on which a Gene-Gene interaction network was generated. The LSCC associated diseases and potential drugs were acquired using Sub-Network Enrichment Analysis. LSCC, lung squamous cell carcinoma.

A detailed description of the SRVS algorithm was produced by Cao et al (15).

Gene expression data. In the present study, two Homo sapiens RNA gene expression datasets [GEO ID: GSE18842 (16) and GSE1987 (17)] were employed to evaluate the classification performance using the LSCC target genes. These datasets were selected from the Illumina BaseSpace Correlation Engine (http://www.illumina.com) and are publicly available at the National Center of Biotechnology Information Gene Expression Omnibus (www.ncbi.nlm.nih.gov/geo/). The data selection criteria were as follows: i) They were human experiments; ii) the data were RNA expression datasets; iii) the data were presented as LSCC case vs. normal control studies; and iv) the tissue was from the lung. From each dataset, expression data of normal controls and patients with LSCC were extracted and used for LSCC case/control classification. Genes of each dataset were limited to LSCC target genes curated within the database LSCC_GD. The key statistics of the two datasets are summarized in Table I.

The gene expression profiles of the two gene expression datasets are also included in LSCC_GD ( LSCC_ $\mathrm{GD} \rightarrow \mathrm{GSE} 18842$ and LSCC_GD $\rightarrow$ GSE1987). Within each dataset, the SRVS-generated weights (SRVSScore) and analysis of variance (ANOVA)-generated P-value score [PValueScore; logic transferred P-values: $-10 x \log (\mathrm{P}$-value)] were also presented. For a given gene, the input of the ANOVA was two vectors of gene expression values, one vector was for the case group subjects and the other was for the control group. The gene expression data were provided in LSCC_GD $\rightarrow$ GSE18842 and LSCC_GD $\rightarrow$ GSE1987. The P-value for a gene was generated from the one-way ANOVA of the case/control comparison using the corresponding expression data. A SRVSScore or a PValueScore represented the significance of a gene to the dataset according to SRVS or ANOVA methods, respectively.

LSCC case/control classification. To identify the best gene vector resulting the highest $\mathrm{CR}$ and the corresponding $\mathrm{CR}$, the LSCC target genes were ranked by SRVSScore in descending order. Subsequently, a Euclidean distance-based multivariate classification (14) was performed for each dataset, followed by a leave-one-out (LOO) cross validation (18). For each run of LOO, the gene expression data of one subject was used for testing and the rest for training. The inputs of the classifier were the expression values of the top $n(n=1,2 \ldots)$ genes, such that the CR of the top $n$ genes could be determined. A permutation of 5,000 runs was then conducted to test the hypothesis, that randomly selected gene sets of the same size can reach equal or higher CR. The permutation P-values (number of runs with equal or better CRs over the number of total runs) were calculated. The gene vector that generated the highest CR was considered the best gene vector, and was selected for the dataset according to the SRVS method.

Following the same process, the best gene vector according to the ANOVA approach was identified for each dataset. For comparison purposes, a CR baseline was also generated using randomly selected gene sets of $\mathrm{n}(n=1,2 \ldots)$ genes. For each point of the CR baseline, the value was the mean of $300 \mathrm{CRs}$, which were from randomly selected genes within the dataset.

\section{Results}

Target genes from LSCC_GD. Due to the lack of space, a small GGI network was presented as an example in Fig. 2. The size of the example network was 7-by-7 (49/260 LSCC target genes). The full GGI network composed of 201/260 LSCC target genes has been presented in the form of adjacency matrix in LSCC_GD $\rightarrow$ GGI Network. An association between two genes indicated that these two genes shared $\geq 1$ pathways (LSCC_GD $\rightarrow$ Related Pathways). There were 59 genes not included in the LSCC associated pathways, and therefore were not presented in the GGI network.

LSCC case/control classification. The maximum CRs are marked at the position of the corresponding number of genes. As depicted in Table II, the results of LOO cross-validation of the two gene ranking method on two datasets were summarized, including the maximum $\mathrm{CRs}$, the corresponding number of top genes and the permutation P-values of the two methods.

Fig. 3 displays the classification results and establishes that compared with the CRs generated by randomly selected gene sets, the genes selected from LSCC target genes by SRVSScore and PValueScore may result in significantly higher classification accuracies. Notably, using only the top genes with the highest SRVSScore/PValueScore, the highest CRs were acquired (Fig. 3 and Table II). These results demonstrated the effectiveness of the selected top genes by SRVS and ANOVA in the differentiation of patients with LSCC from controls, and those selected genes for a specific patient with LSCC group should be the main genetic targets for the prognosis 
Table II. Leave-one-out cross validation and permutation results.

GSE18842 (case/control: 46/45)

GSE1987 (case/control: 17/9)

\begin{tabular}{|c|c|c|c|c|}
\hline \multirow[b]{2}{*}{ Analysis results } & & \\
\hline & SRVS & ANOVA & SRVS & ANOVA \\
\hline Maximum CR & 100.00 & 98.90 & 100.00 & 96.15 \\
\hline \#Selected Genes & 17 & 24 & 10 & 2 \\
\hline P-value & $5.0 \times 10^{-4}$ & $4.9 \times 10^{-2}$ & $1.8 \times 10^{-3}$ & $2.0 \times 10^{-3}$ \\
\hline Unique genes from all datasets (\%) & $94.11 \%(16 / 17)$ & $91.67 \%(22 / 24)$ & $90.00 \%(9 / 10)$ & $0.00 \%(0 / 2)$ \\
\hline Overlapping genes of two methods (\%) & $23.53 \%(4 / 17)$ & $16.67 \%(4 / 24)$ & $10.00 \%(1 / 10)$ & $50.00 \%(1 / 2)$ \\
\hline
\end{tabular}

ANOVA, analysis of variance; CR, classification ratio; SRVS, sparse representation-based variable selection.

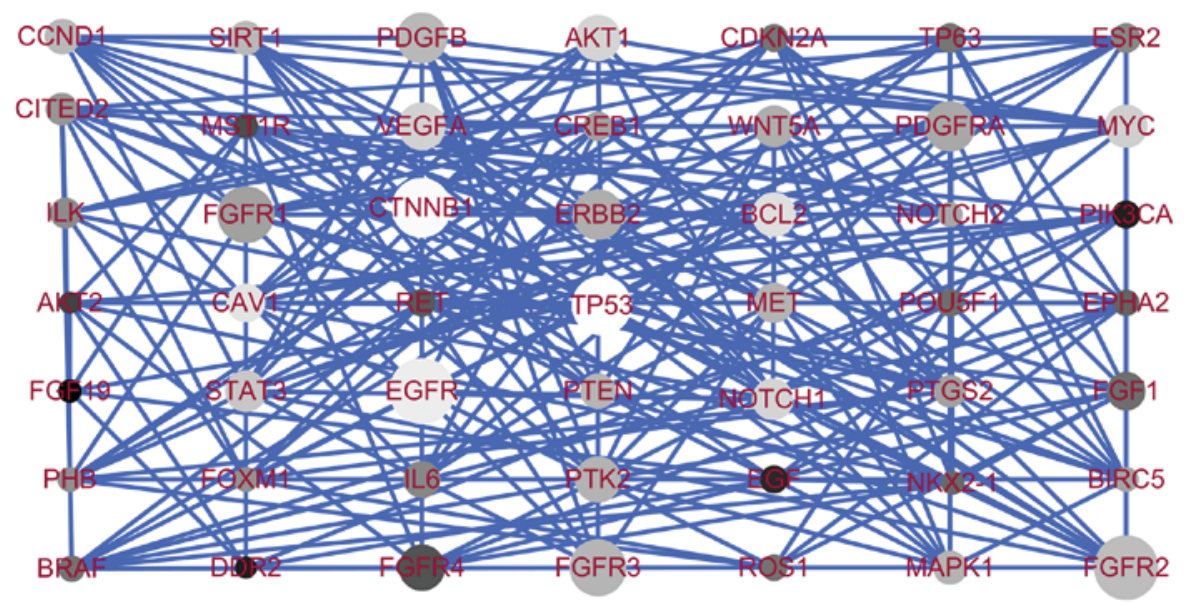

Figure 2. The Gene-Gene Interaction Network composed of the 49/260 LSCC target genes from LSCC_GD. The edge weight between two node/genes represents the number of pathways shared by the two genes. The larger the size of a node, the higher the number of pathways (LSCC_GD $\rightarrow$ Related Pathways) that include this gene. The brighter the color, the higher the Fisher's centrality of the gene (number of other genes connected). The adjacency matrix is presented in the LSCC_GD GGI Network. LSCC, lung squamous cell carcinoma.

and treatment of the patients in the group. Additionally, as displayed in Table II, the SRVS method outperformed ANOVA method in terms of CR in all datasets $\left(5.0 \times 10^{-4} \mathrm{vs} .4 .9 \times 10^{-2}\right.$ and $1.8 \times 10^{-3}$ vs. $2.0 \times 10^{-3}$ for GSE18842 and GSE1987, respectively).

Table II also depicts that, for each dataset, the top genes selected by each method may be significantly different. For the SRVS method, the unique genes selected for the two datasets ranged from $90-94.11 \%$. For the ANOVA method, the two dataset (GSE18842 and GSE1987) demonstrated 91.67 and $0 \%$ unique genes, respectively [Table II $\rightarrow$ Unique genes from all datasets (\%)]. These results indicated the group specificity of the genome variation of the patients within the two datasets.

Notably, the optimum gene markers for distinct datasets selected by SRVS and ANOVA may be considerably different (Table II). The genes selected by the SRVS method demonstrated a $<23.53 \%$ overlap with that of the ANOVA method for both datasets [Table II $\rightarrow$ Overlap genes of two methods (\%)]. The results indicated that SRVS performs differently and more effectively than ANOVA.

\section{Discussion}

LSCC is an aggressive cancer type, and the overall prognosis for patients with LSCC is poor. Previously, numerous molecular therapy targeted studies have been conducted $(19,20)$, with hundreds of risk genes identified for the disease. The majority of these genes serve roles within LSCC-associated genetic pathways, and a number of them were indicated as drug targets for the disease, such as FGFR1 and discoidin domain receptor tyrosine kinase 2 (DDR2) (20-22).

Only a limited number of genes have been frequently detected in LSCC cases. For instance, focal FGFR1 amplification occurs in up to $22 \%$ of LSCC cases (21). FGFR1 amplification in cells is dependent on FGFR signaling and is sensitive to FGFR inhibitors (21). Somatic mutations in the DDR2 kinase gene were also indicated as the potential targets for the treatment of a portion of patients with $\operatorname{LSCC}(20,22)$. These results reflected the heterogeneity of human tumor types (23), and explain the large size of the LSCC risk gene pool curated through previous studies (4-12).

Whilst novel LSCC genes are being actively investigated in continuous genetic and genomic studies performed, significantly less studies have been conducted to test the validity of the existing LSCC risk genes as a whole for their diagnostic and predictive capabilities for LSCC. We hypothesized that, if the current LSCC gene pool is sufficient to cover the majority of the genes underlying the genetic pathogeneses of LSCC, then for a given group of patients 
A

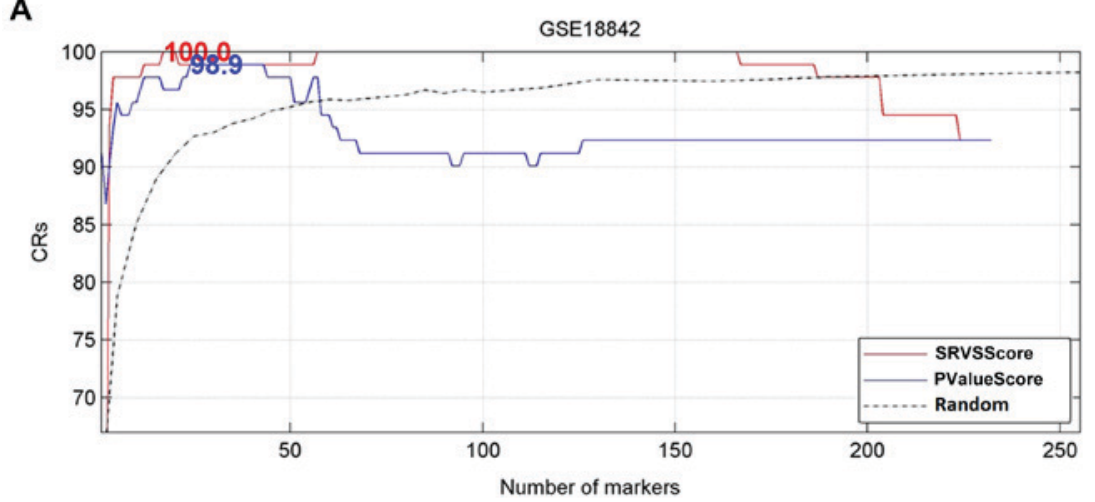

B

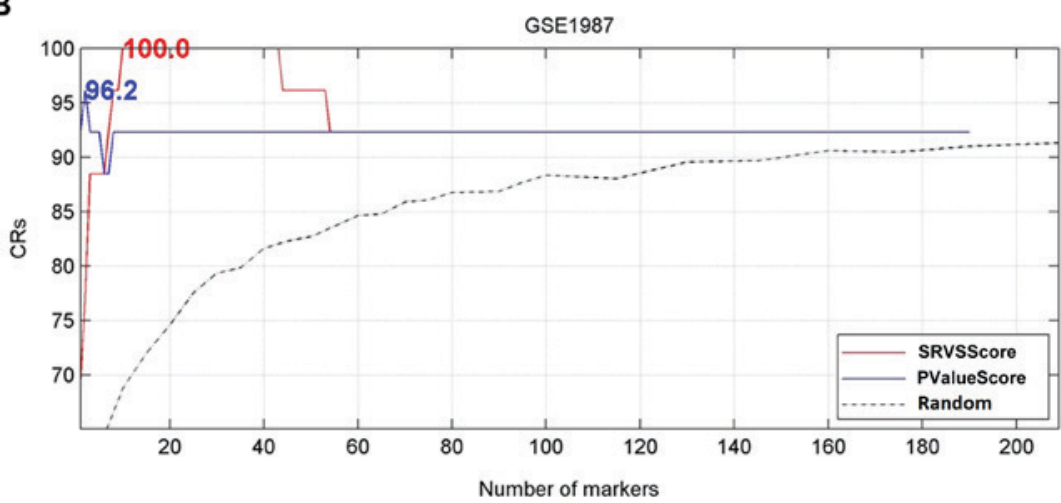

Figure 3. Comparison of different metrics through a leave-one-out crosses validation. Genes were ranked in ascending order according to SRVSScore or PValueScore for SRVS or analysis of variance method, respectively. SRVS, sparse representation-based variable selection; CR, classification ratio; SRVSScore, SRVS-generated weights; PValueScore, analysis of variance-generated P-value score.

with LSCC, $\geq 1$ gene vector from the LSCC gene pool exists that possesses significance in the classification/prediction of patients with LSCC from controls. If this hypothesis were true, then another issue would arise, in how the optimum gene combination from the target pool for a specific patient group would be identified.

To test this hypothesis, comprehensive literature data mining using Pathway Studio was conducted, which identified 260 LSCC target genes. Pathway Studio covers $>40,000,000$ scientific papers. Each association between these genes and LSCC was supported by $\geq 1$ literature reports (LSCC_GD $\rightarrow$ Ref for Disease-Gene Relation). Within LSCC_GD, there are also 100 pathways (LSCC_GD $\rightarrow$ Related Pathways), 101 disease-subnetworks (LSCC_GD $\rightarrow$ Diseases) and 105 potential drugs/small molecules (LSCC_GD $\rightarrow$ Potential Drugs) present when these genes were significantly enriched.

PEA demonstrated that the majority of these genes (201/260) were significantly enriched within multiple genetic pathways implicated in LSCC $\left(\mathrm{P}<4.4 \times 10^{-5}\right.$; $\mathrm{q}=0.001$ for FDR). For instance, there are 66 genes significantly enriched within four cell apoptosis pathways $\left(\mathrm{P}<3.0 \times 10^{-7} ; \mathrm{q}=0.001\right.$ for FDR) $(24,25)$, including negative regulation of the apoptotic process [Gene Ontology (GO): 0006916; $\mathrm{P}=1.1 \times 10^{-14}$; the apoptotic process (GO: 0008632; $\mathrm{P}=9.9 \times 10^{-08}$ ); positive regulation of the apoptotic process (GO: 0043065; $\mathrm{P}=2.2 \times 10^{-7}$ ); and negative regulation of cysteine-type endopeptidase activity involved in the apoptotic process (GO: 0043154; $\mathrm{P}=3.0 \times 10^{-7}$ ). There were also 79 genes significantly enriched within 9 pathways associated with cell growth and proliferation
$\left(\mathrm{P}<2.8 \times 10^{-5}\right)(26)$ and 72 genes enriched within protein kinases $\left(\mathrm{P}<3.7 \times 10^{-6}\right)(27,28)$. More pathways can be identified from LSCC_GD $\rightarrow$ Related Pathways.

Disease SNEA demonstrated that, 250/260 genes were significantly overlapped with the risk genes of 101 diseases $\left(\mathrm{P}<1.9 \times 10^{-33} ; \mathrm{q}=0.001\right.$ for FDR $)$. The majority of these 101 diseases are different cancers types, and a number of them were associated with LSCC, including gastric (29) and breast cancer (30). More results from SNEA can be identified at LSCC_GD $\rightarrow$ Related Diseases.

Within LSCC_GD, there were 56 known LSCC drugs (LSCC_GD $\rightarrow$ Related Drugs) that underwent clinical trials and demonstrated effectiveness in treating LSCC. These 56 drugs demonstrated significant overlap (13 overlapped drugs; $\left.\mathrm{P}=1.31 \times 10^{-18}\right)$ with the top 105 potential drugs/small molecules (LSCC_GD $\rightarrow$ Potential Drugs), whose gene sub-networks were significantly enriched with the 260 LSCC genes. Additionally, a number of the 260 LSCC genes were target genes of known LSCC drugs. For instance, AZD4547, a FGFR inhibitor, is a key drug in the treatment of LSCC. This may be explained due to AZD4547 exhibiting a highly selective profile across a lung cell line panel, potently inhibiting cell growth only in those lines harboring amplified FGFR1 (31). These results demonstrated that the 260 LSCC genes were associated with LSCC and therefore may possess classification/prediction power for the disease; however, due to the heterogeneity of LSCC and the specificity of human genome variation (13), the significance of these genes being used as markers for disease diagnosis and personalized treatment still requires testing. 
To address this issue, LSCC case/control classification was conducted on two independent gene expression datasets, with two algorithms (SRVS and ANOVA) for gene selection from the 260 LSCC genes. The basic theory for gene selection is that mutations of these 260 genes will not be exhibited in a number of patients with LSCC, and therefore are not effective as biomarkers for all patients.

Compared with randomly selected genes, those selected by SRVS and ANOVA generated significantly higher predication power (permutation $\mathrm{P}<1.8 \times 10^{-3}$ for SRVS, and $\mathrm{P}<5.0 \times 10^{-2}$ for ANOVA), with improved high classification accuracy (SRVS vs. ANOVA: $100 \%$ vs. $98.9 \%$, and $100 \%$ vs. $96.15 \%$, for datasets GSE18842 and GSE1987, respectively), as depicted in Table II. These results indicated that, for a given dataset, a gene vector, from the 260 LSCC gene pools, that could be used as biomarker vector for the diagnosis and prognosis of the disease exists. Notably, SRVS outperforms ANOVA in terms of CR on both datasets. This indicated the effectiveness of the SRVS method for feature selection. It is notable that both datasets used LSCC lung tissue as sample source, which may partially explain the high classification results of both methods.

Cross analysis on the genes selected demonstrated that optimum biomarkers are dataset specific, as depicted in Table II. These results indicated the specificity of the genomic variations of different patients (12), and highlighted the necessity of genomic variable selection in the diagnosis and treatment of patients with LSCC.

To conclude, the present study indicated that the 260 curated LSCC genes from previous studies demonstrated a strong association with the pathogenesis of LSCC, and possessed significant diagnostic power as a biomarker network. Furthermore, SRVS is an effective method to select the optimum gene sub-set for personalized diagnosis and treatment for a specific group of patients with LSCC.

\section{Acknowledgements}

Not applicable.

\section{Funding}

The present study is partially supported by non-small cell lung cancer research funding of Jiangsu Provincial Planning Commission, clinical diagnosis and treatment of small lung lesions and normative research of Wuxi City Health Planning Commission (grant no. MS201625) and 2017 Fifth Provincial '333 Project' Research Project.

\section{Availability of data and materials}

All data generated or analyzed during the present study are included in this published article.

\section{Authors' contributions}

$\mathrm{BH}$ and $\mathrm{NZ}$ contributed to the design and data collection of the study, HC and GY contributed to the data collection and analysis. All authors contributed to the writing and revising of the manuscript.

\section{Ethics approval and consent to participate}

Not applicable.

\section{Patient consent for publication}

Not applicable.

\section{Competing interests}

The authors declare that they have no competing interests.

\section{References}

1. Heist RS, Mino-Kenudson M, Sequist LV, Tammireddy S, Morrissey L, Christiani DC, Engelman JA and Iafrate AJ: FGFR1 amplification in squamous cell carcinoma of the lung. J Thorac Oncol 7: 1775-1780, 2012.

2. American Cancer Society: Cancer Facts and Figures 2018. Atlanta, Ga: American Cancer Society, 2018. Available online. Last accessed April 27, 2018.

3. Molina JR, Yang P, Cassivi SD, Schild SE and Adjei AA: Non-small cell lung cancer: Epidemiology, risk factors, treatment, and survivorship. Mayo Clin Proc 83: 584-594, 2008.

4. Alberg AJ, Brock MV and Samet JM: Epidemiology of lung cancer: Looking to the future. J Clin Oncol 23: 3175-3185, 2005.

5. Shigematsu H, Lin L, Takahashi T, Nomura M, Suzuki M, Wistuba II, Fong KM, Lee H, Toyooka S, Shimizu N, et al: Clinical and biological features associated with epidermal growth factor receptor gene mutations in lung cancers. J Natl Cancer Inst 97: 339-346, 2005.

6. Lynch TJ, Bondarenko I, Luft A, Serwatowski P, Barlesi F, Chacko R, Sebastian M, Neal J, Lu H, Cuillerot JM and Reck M: Ipilimumab in combination with paclitaxel and carboplatin as first-line treatment in stage IIIB/IV non-small-cell lung cancer: Results from a randomized, double-blind, multicenter phase II study. J Clin Oncol 30: 2046-2054, 2012.

7. Gibbons DL, Byers LA and Kurie JM: Smoking, p53 mutation, and lung cancer. Mol Cancer Res 12: 3-13, 2014.

8. Stilgenbauer S, Schnaiter A, Paschka P,Zenz T, Rossi M,Döhner K, Bühler A, Böttcher S, Ritgen M, Kneba M, et al: Gene mutations and treatment outcome in chronic lymphocytic leukemia: Results from the CLL8 trial. Blood 123: 3247-3254, 2014.

9. Rosenfeld MR, Malats N, Schramm L, Graus F, Cardenal F, Viñolas N, Rosell R, Torà M, Real FX, Posner JB and Dalmau J: Serum anti-p53 antibodies and prognosis of patients with small-cell lung cancer. J Natl Cancer Inst 89: 381-385, 1997.

10. Behnam AzadB,Lisok A, Chatterjee S, Poirier JT,Pullambhatla M, Luker GD, Pomper MG and Nimmagadda S: Targeted imaging of the atypical chemokine receptor 3 (ACKR3/CXCR7) in human cancer xenografts. J Nucl Med 57: 981-988, 2016.

11. Zheng CX, Gu ZH, Han B, Zhang RX, Pan CM, Xiang Y, Rong XJ, Chen X, Li QY and Wan HY: Whole-exome sequencing to identify novel somatic mutations in squamous cell lung cancers. Int J Oncol 43: 755-764, 2013.

12. Quintanal-Villalonga Á, Carranza-Carranza A, Meléndez R, Ferrer I, Molina-Pinelo S and Paz-Ares L: Prognostic role of the FGFR4-388Arg variant in lung squamous-cell carcinoma patients with lymph node involvement. Clin Lung Cancer 18: 667-674.e1, 2017.

13. Lu YF, Goldstein DB, Angrist M and Cavalleri G: Personalized medicine and human genetic diversity. Cold Spring Harb Perspect Med 4: a008581, 2014.

14. Lorenzi PL, Claerhout S, Mills GB and Weinstein JN: A curated census of autophagy-modulating proteins and small molecules: Candidate targets for cancer therapy. Autophagy 10: 1316-1326, 2014.

15. Cao H, Duan J, Lin D, Shugart YY, Calhoun V and Wang YP: Sparse representation based biomarker selection for schizophrenia with integrated analysis of fMRI and SNPs. Neuroimage 102: 220-228, 2014

16. Sanchez-Palencia A, Gomez-Morales M, Gomez-Capilla JA, Pedraza V, Boyero L, Rosell R and Fárez-Vidal ME: Gene expression profiling reveals novel biomarkers in nonsmall cell lung cancer. Int J Cancer 129: 355-364, 2011. 
17. Dehan E, Ben-Dor A, Liao W, Lipson D, Frimer H, Rienstein S, Simansky D, Krupsky M, Yaron P, Friedman E, et al: Chromosomal aberrations and gene expression profiles in non-small cell lung cancer. Lung Cancer 56: 175-184, 2007.

18. Kohavi R: A study of cross-validation and bootstrap for accuracy estimation and model selection. Proceedings of the Fourteenth International Joint Conference on Artificial Intelligence. San Mateo, CA: Morgan Kaufmann 2: 1137-1143, 1995.

19. Gan TQ, Xie ZC, Tang RX, Zhang TT, Li DY, Li ZY and Chen G: Clinical value of miR-145-5p in NSCLC and potential molecular mechanism exploration: A retrospective study based on GEO, qRT-PCR, and TCGA data. Tumour Biol 39: 1010428317691683 , 2017.

20. Hammerman PS, Sos ML, Ramos AH, Xu C, Dutt A, Zhou W, Brace LE, Woods BA, Lin W, Zhang J, et al: Mutations in the DDR2 kinase gene identify a novel therapeutic target in squamous cell lung cancer. Cancer Discov 1: 78-89, 2011.

21. Weiss J, Sos ML, Seidel D, Peifer M, Zander T, Heuckmann JM, Ullrich RT, Menon R, Maier S, Soltermann A, et al: Frequent and focal FGFR1 amplification associates with therapeutically tractable FGFR1 dependency in squamous cell lung cancer. Sci Transl Med 2: 62ra93, 2010.

22. Sos ML and Thomas RK: Genetic insight and therapeutic targets in squamous-cell lung cancer. Oncogene 31: 4811-4814, 2012.

23. Loeb LA, Loeb KR and Anderson JP: Multiple mutations and cancer. Proc Natl Acad Sci USA 100: 776-781, 2003.

24. Ramsey MR, Wilson C, Ory B, Rothenberg SM, Faquin W, Mills AA and Ellisen LW: FGFR2 signaling underlies p63 oncogenic function in squamous cell carcinoma. J Clin Invest 123: $3525-3538,2013$
25. Ling B and Wei GZ: p53: Structure, function and therapeutic applications. J Cancer Mol 2: 141-153, 2006.

26. Courtney KD, Corcoran RB and Engelman JA: The PI3K pathway as drug target in human cancer. J Clin Oncol 28: 1075-1083, 2010.

27. Yip PY: Phosphatidylinositol 3-kinase-AKT-mammalian target of rapamycin (PI3K-Akt-mTOR) signaling pathway in non-small cell lung cancer. Transl Lung Cancer Res 4: 165-176, 2015.

28. Davies H, Hunter C, Smith R, Stephens P, Greenman C Bignell G, Teague J, Butler A, Edkins S, Stevens C, et al: Somatic mutations of the protein kinase gene family in human lung cancer. Cancer Res 65: 7591-7595, 2005.

29. Kurishima K, Satoh H, Kagohashi K, Homma S, Nakayama H, Ohara G, Ishikawa $\mathrm{H}$ and Hizawa N: Patients with lung cancer with metachronous or synchronous gastric cancer. Clin Lung Cancer 10: 422-425, 2009.

30. Genç B, Solak A, Sahin N and Gülşen A: Metastasis to the male breast from squamous cell lung carcinoma. Case Rep Oncol Med 2013: 593970, 2013

31. Zhang J, Zhang L, Su X, Li M, Xie L, Malchers F, Fan S, Yin X, Xu Y, Liu K, et al: Translating the therapeutic potential of AZD4547 in FGFR1-amplified non-small cell lung cancer through the use of patient-derived tumor xenograft models. Clin Cancer Res 18: 6658-6667, 2012.

This work is licensed under a Creative Commons Attribution-NonCommercial-NoDerivatives 4.0 International (CC BY-NC-ND 4.0) License. 